\title{
Ethnobiological Study of Svaneti Fungi and Lichens: History of Research, Diversity, Local Names and Traditional Use
}

\author{
I. Kupradze 1, ", A. Jorjadze', A. Arabidze', T. Beltadze', K. Batsatsashvili \\ N. Y. Paniagua Zambrana ${ }^{3}$, R. W. Bussmann ${ }^{4}$ \\ ${ }^{1}$ Institute of Botany of IliaStateUniversity, Tbilisi, Georgia \\ ${ }^{2}$ Institute of Ecology of Ilia State University, Tbilisi, Georgia \\ ${ }^{3}$ Herbario Nacional de Bolivia, Universidad Mayor de San Andres, La Paz, Bolivia \\ ${ }^{4}$ William L. Brown Center, Missouri Botanical Garden, St. Louis, USA \\ Email address: \\ Inga.Kupradze@gmail.com (I. Kupradze)
}

\section{To cite this article:}

I. Kupradze, A. Jorjadze, A. Arabidze, T. Beltadze, K. Batsatsashvili, N. Y. Paniagua Zambrana, R. W. Bussmann. Ethnobiological Study of Svaneti Fungi and Lichens: History of Research, Diversity, Local Names and Traditional Use. American Journal of Environmental Protection. Special Issue: Applied Ecology: Problems, Innovations.Vol. 4, No. 3-1, 2015, pp. 101-110. doi: 10.11648/j.ajep.s.2015040301.26

\begin{abstract}
Research about macro-fungi of Svaneti, a historical province of Georgia, started in the $1920^{\text {th }}$ with a botanical expedition of R. Singer, under the auspices of the National Museum of Vienna. At present about 400 species of macro-fungi are known for Svaneti, 67 of which are edible (and used for food in Georgia), and 27 are poisonous. The history of Svaneti lichen research started with analysis of the material collected by H. Lojka and M. Dechy in 1884-1885 made by E. Vainio. As of now, 250 species of lichens are known for the region. In the present study ethnobiological interviews on Svaneti macro-fungi and lichens were conducted in 16 villages of Svaneti. Our analysis shows that the local population uses mainly 22 species of mushrooms for food, 10 of which are used most frequently: Armillaria mellea (Vahl) P. Kumm., Agaricus arvensis Schaeff., A. campestris L., Cantharellus cibarius Fr., Lactarius piperatus (L.) Pers., L. deliciosus (L.) Gray, Macrolepiota excoriata (Schaeff.) Wasser, M. procera (Scop.) Singer, Ramaria flava (Schaeff.) Quel. A comparison of the vernacular names given in the literature and mentioned in the interviews collected during the present study shows that the population of Svaneti must have had better knowledge of mushrooms in the past. This is evidenced by a much higher number of local names in literature: of about 50 vernacular names of particular species or groups of fungi found in the literature, only 12 are still used by the population. This paper presents various uses of macro-fungi in Svaneti as quoted in literature and based on interview data. Lichens, particularly, Usnea and Bryoria species, as well as Ramalina thrausta (Ach.) Nyl.are called "Pimpa" or "Pimpous" and "Chach". The only use of these species was by hunters as field-bedding to keep warm in the cold winters. In addition, we describe in the present paper how fungi and lichen uses were reflected in the local folklore.
\end{abstract}

Keywords: Macro-Fungi, Lichens, Ethnobiology, Georgia, Caucasus

\section{Introduction}

The first data on fungi of Svaneti, a historical province of Georgia, were published by R. Singer [1], who visited Svaneti in 1929 within the framework of a botanical expedition organized by the National Museum of Vienna. His paper provides a list of 95 species of macro-fungi found in Svaneti. Later the mycological investigation of the province was continued by I. Nakhutsrishvili (Agaricales s.1.) [2], V. Gulmagarashvili (Polyporales) [3], T. Anchabadze
(Discomycetes) [4]. Based on current literature (mainly, [5]), and herbarium data, about 400 species of macro-fungi are known from Svaneti at present, of which 67 are edible (used for food in Georgia) and 27 are poisonous.

The history of Svaneti lichen research started with [6], listing 76 taxa, based on the analysis of the material collected by H. Lojka and M. Dechy in 1884-1885. Reference [7] mentions 26 taxa based on collections by S. Sommier and E. Levier. Reference [8] mentions a single species from material collected by F. Filarszky, reference [9] - two species, and reference [10] - 56 taxa identified from the collection of D. 
Sosnovsky. Later research was continued by Tz. Inashvili in 1975-1977. At present 250 species of lichens are known from Svaneti.

No specific studies were, however conducted on the ethnobiology of the fungi (macro-fungi) and lichens of Svaneti and generally Georgia so far. However, vernacular names with descriptions of fungi and in some cases their use are present in a number of dictionaries [11-17].

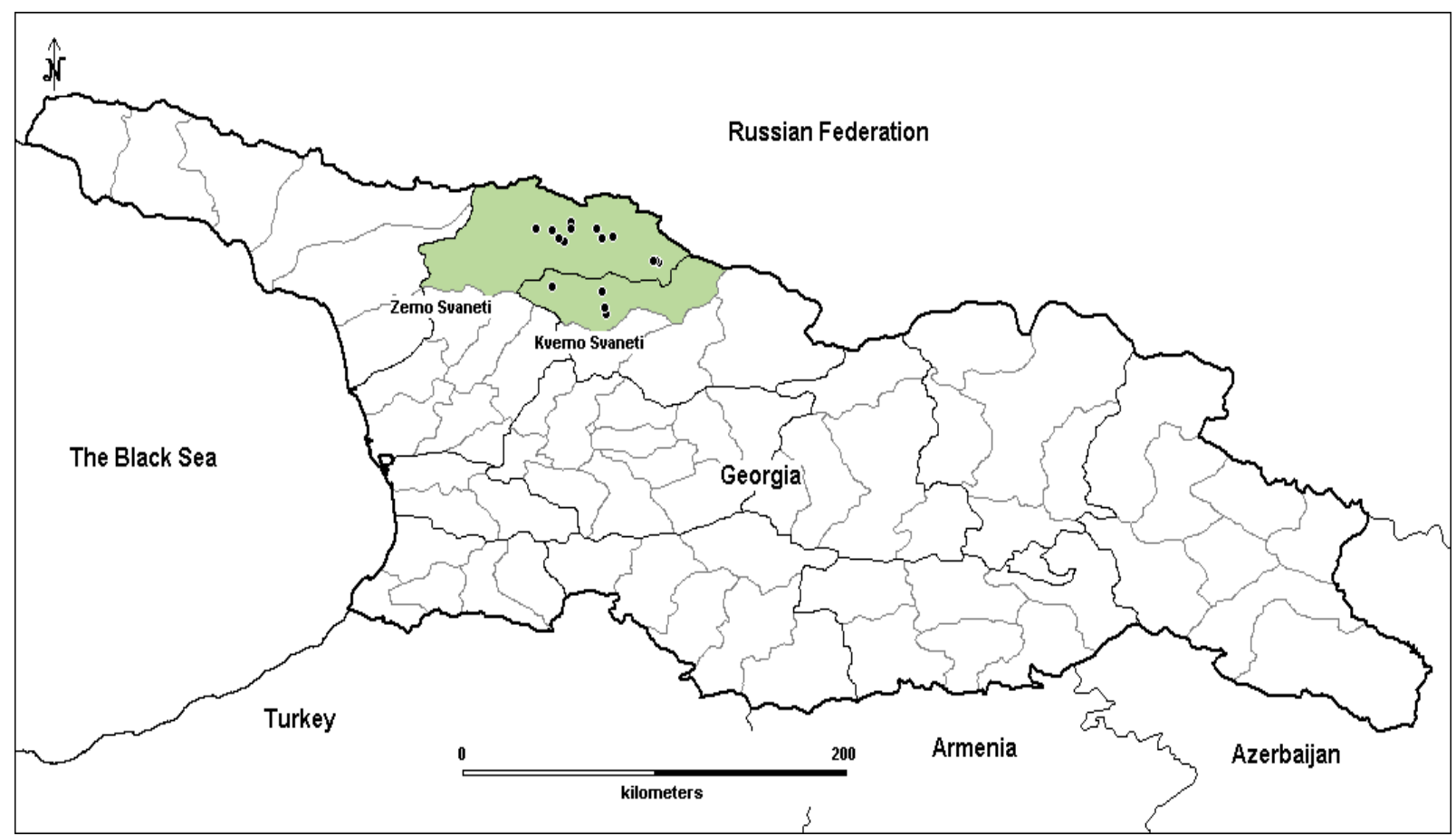

Figure 1. Schematic map of the study area. The territory of Svaneti is colored and the villages where the interviews were collected are shown as black circles.

\section{Study Area}

\subsection{Location}

Svaneti is a historical province of Georgia, located on the south-facing macro-slope of the western part of the Greater Caucasus. Svanetis Kavkasioni, a part of the Main Watershed Range of the Greater Caucasus, creates the natural northern border of Svaneti between Gvandra and Mt. Pasismta. From the west the region is bordered by the Kodori and Akibo ranges, from the south-west by the rivers Bordjali and Gandishis-Ghele, both tributaries of the river Enguri, as well as by the Bokunstha range. From the east Svaneti is bordered by the northern part of Lechkhumi range, and from the south by the southern part of the same Lechkhumi range, and the eastern part of Egrisi range. The Svaneti range divides the region into two depressions: Zemo (Upper) Svaneti and Kvemo (Lower) Svaneti creating a watershed between the Enguri and Tskhenistskali basins. The region has an altitudinal gradient from $800-4,500 \mathrm{~m}$ a.s.1. [18, 19].

\subsection{Administrative Division}

Administratively Zemo Svaneti belongs to Mest'ia municipality (with an area of $3,045 \mathrm{~km}^{2}$ ), which is part of the Samegrelo-Zemo Svaneti Region (Mkhare) and includes Mest'ia, as its administrative center, and 134 villages of 16 communes: Becho, Ch'uberi, Etseri, Idliani, Ipari, Kala, Khaishi, Latali, Lakhamula, Lenjeri, Mulakhi, Nakra, Pari,
Tskhumari, Ts'virmi, and Ushguli. Kvemo Svaneti is part of Lentekhi municipality (with an area of $1,344 \mathrm{~km}^{2}$ ) and belongs to the Racha-Lechkhumi-Kvemo Svaneti Region (Mkhare). It includes the settlement Lentekhi as administrative center, and 59 villages of seven communes: Chikhareshi, Choluri, Kheledi, Khopuri, Rtskhmeluri, Tsana, and Zhakhunderi [20] (fig. 1).

Svaneti is a historical province of Georgia, located on the south-facing macro-slope of the western part of the Greater Caucasus. Svanetis Kavkasioni, a part of the Main Watershed Range of the Greater Caucasus, creates the natural northern border of Svaneti between Gvandra and Mt. Pasismta. From the west the region is bordered by the Kodori and Akibo ranges, from the south-west by the rivers Borjali and Gandishis-Ghele, both tributaries of the river Enguri, as well as by the Boq'unsta range. From the east Svaneti is bordered by the northern part of Lechkhumi range, and from the south by the southern part of the same Lechkhumi range, and the eastern part of Egrisi range. The Svaneti range divides the region into two depressions: Zemo (Upper) Svaneti and Kvemo (Lower) Svaneti creating a watershed between the Enguri and Tskhenists'q'ali basins. The region has an altitudinal gradient from $800-4,500 \mathrm{~m}$ a.s.1. [18, 19].

\subsection{Climate}

The whole western Greater Caucasus experiences the influence of the Black Sea and moist westerly winds. The mean temperature of the warmest months (July-August) in 
Svaneti decreases from $+22^{\circ} \mathrm{C}$ at relatively low altitudes to $+7^{\circ} \mathrm{C}-1^{\circ} \mathrm{C}$ above 3,200 $\mathrm{m}$ a.s.l.; and the mean temperature of the coldest month (January) from $+10^{\circ} \mathrm{C}$ to $-30^{\circ} \mathrm{C}-35^{\circ} \mathrm{C}$. The annual precipitation is $1,500-2,000 \mathrm{~mm}$ [21].

\subsection{Vegetation Cover}

The vegetation of the region includes montain forest, subalpine, alpine, subnival and nival zones and corresponds to the West Caucasian, i.e. Colchic, type of the vegetation vertical zonation $[19,22]$.

\subsection{Population and Local Language}

The region has a population of about 24,000 people; $99 \%$ of the population are Georgians [20]. Most inhabitants speak both Georgian and Svanetian. The Svanetian language belongs to the Kartvelian ${ }^{1}$ group of the Iberian-Caucasian family of languages (there are several Kartvelian languages: Georgian, Zanian (Megrelian, Chanian) and Svanetian), and Svanetian is considered an old branch of the Proto-Kartvelian language. Nevertheless, it still has common basics and grammar structures with Georgian-Zanian languages; archaisms conserved in Svanetian are very important for linguistics. Svanetian does not have written language historically the common colloquial language for all the Georgian tribes as well as formal language in the Georgian State(s) was Georgian - but rich Svanetian folklore provides sufficient material for linguistic research. The Svanetian language has four dialects: Balszemouri and Balskvemouri in Zemo Svaneti, and Lashkhuri and Lentekhuri in Kvemo Svaneti. In Kvemo Svaneti some researchers distinguish the third dialect, Choluruli, which is very close to Lashkhuri. Each of the listed dialects is only used in small groups of villages in Svaneti [23].

\section{Methods}

Research was conducted in July 2014. After obtaining prior informed consent for interviews, semi-structured interviews were conducted with participants in local villages.

Vernacular names provided by the respondents were analyzed in parallel to the names given in the dictionaries listed above.

Georgian names are transliterated according to a Romanization system for Georgian approved by US Board on Geographic Names (BGN)/Permanent Committee on Geographical Names (PCGN) 2009 Agreement.

Fungi and lichen species mentioned in the present paper are documented by collections stored at TBI; collection numbers of specimens from Svaneti are given in table 1 for each species. Species nomenclature follows [24].

\section{Results and Discussion}

Ethnobiological interviews were collected in 16 villages of

\footnotetext{
1"Kartveli" means "the Georgian" in the Georgian language
}

Zemo Svaneti (Mest'ia, Ebut'i, Ieli, Labsq'aldi, Lakhamula, Mazeri, Tskhek'vani, Ts'virmi, T'viberi, Ushguli: Zhvibiani, Chvibiani and Murq'meli); and Kvemo Svaneti (Babili, Gvimbrala, Khopuri, Mananauri) (fig. 1).

\subsection{Fungi}

\subsubsection{Mushrooms Used for Food}

According to literature $[2,5,25,26]$ and herbarium data, 67 species of mushrooms use for food in Georgia occur in Svaneti (Table 1). Our analysis of interviews collected in Svaneti shows that the local population uses mainly 22 species of mushrooms for food, 10 of which are used most frequently: Armillaria mellea (Vahl) P. Kumm., Agaricus arvensis Schaeff., A campestris L., Cantharellus cibarius Fr., Lactarius piperatus (L.) Pers., L. deliciosus (L.) Gray, Macrolepiota excoriata (Schaeff.) Wasser, M. procera (Scop.) Singer, Ramaria flava (Schaeff.) Quel. Morphologically similar species are often referred to by the same vernacular name, e.g. mushrooms morphologically close to Boletus s.l. are all called "Khortsa sok'o" or "Duma sok'o", Agaricus spp. as well as Macrolepiota spp. are called "Kama sok'o".

The local population interviewed recognized mushrooms to different degrees: $90-100 \%$ of the respondents recognized and used Armillaria mellea and Lactarius piperatus; 70-80\% recognized and used Agaricus arvensis, A. campestris, Cantharellus cibarius, Macrolepiota excoriata, M. procera, Ramaria flava, Lactarius deliciosus; 30-60\% - Amanita caesarea (Scop.) Pers., Pleurotus cornucopiae (Paulet) Rolland, P. ostreatus (Jacq.) P. Kumm., Polyporus squamosus (Huds.) Fr., Boletus s.1., and less than $25 \%$ - Coprinopsis atramentaria (Bull.) Redhead, Vilgalys \& Moncalvo, Bovista spp., Calvatia spp., Lycoperdon spp., etc. Collectors paid major attention to the fruit-body shape, color and smell. They also considered brightly colored and / or pungent smelling mushrooms as well as unusually shaped ones as suspicious. Almost all mushroom species with white caps were considered "Kama sok'o", which is the major Georgian name for Agaricus species. This perception of local collectors often causes confusion of Agaricus with Amanita species, which leads to poisoning incidents with frequent lethal outcome. In some cases the death of whole families was mentioned by the respondents. Local custom responded to this problem by elaborating a kind of "safety test" for mushrooms when eating them and this custom is described in the local folklore: the mushroom dish was first eaten by the oldest member of a family together with a glass of vodka. If the mushroom appeared safe, the dish was then eaten by the other members of the family. This "test" unfortunately does nothing to avoid Amanita poisoning, which might manifest itself only days after ingesting the mushrooms. Armillaria mellea and Lactarius piperatus were considered the safest among the fungi. These species were both eaten freshly collected, and dried or salted (by an ordinary technique) for winter use. In Svaneti mushrooms are mainly roasted, stewed or used for "K'ubdari", filling; soups are not prepared of mushrooms. Caps of some species, e.g. Lactarius deliciosus and Macrolepiota spp. were eaten raw by

\footnotetext{
${ }^{2}$ "K'ubdari" is a Svanetian pie with beef filling.
} 
shepherds.

Table 1. Edible Fungi Occurring in Svaneti

\begin{tabular}{|c|c|c|c|c|c|}
\hline Scientific Name (Collection Nos.) & $\begin{array}{l}\text { Major Georgian } \\
\text { names according to } \\
\text { the literature } \\
\end{array}$ & $\begin{array}{l}\text { Names } \\
\text { mentioned in } \\
\text { the interviews }\end{array}$ & $\begin{array}{l}\text { Numberof res- } \\
\text { pondents who } \\
\text { mentioned the species }\end{array}$ & Season & Habitat; Substrate \\
\hline $\begin{array}{l}\text { Agaricus arvensis Schaeff.* } \\
\text { (TBI5010029) }\end{array}$ & Mindvris kama & Kama & 72 & Jul-Oct & Meadows;Manure, soil \\
\hline $\begin{array}{l}\text { Agaricus campestris L.* } \\
\text { (TBI5010030) }\end{array}$ & Mdelos kama & Kama & 72 & Jul-Oct & Grassy areas;Manure, soil \\
\hline $\begin{array}{l}\text { Agaricus silvaticus Schaeff. } \\
\text { (TBI5010031) }\end{array}$ & Tq'is kama & & 0 & Aug-Sep & Coniferous forest; Soil \\
\hline $\begin{array}{l}\text { Amanita caesarea (Scop.) Pers.* } \\
\text { (TBI5009282) }\end{array}$ & Niq'vi & Neq'v & 28 & Jul-Oct & Primarily oak forest; Soil \\
\hline Amanita fulva Fr. (TBI5009283) & Ts'ablisperi livliva & & 0 & May-Oct & Forest; Soil \\
\hline $\begin{array}{l}\text { Amanita rubescens Pers. } \\
\text { (TBI5009310) }\end{array}$ & Marts'q'vio & & 0 & Jul-Oct & Forest; Soil \\
\hline $\begin{array}{l}\text { Amanita vaginata (Bull.) Lam. } \\
\text { (TBI5009296) }\end{array}$ & Rukhi livliva & & 0 & Jul-Oct & Forest; Soil \\
\hline $\begin{array}{l}\text { Armillaria mellea (Vahl) P.Kumm.* } \\
\text { (TBI5009311) }\end{array}$ & Manch'k'vala & Manch'k'varai & 85 & May-Nov & $\begin{array}{l}\text { Primarily beech forest; } \\
\text { Trunks and stumps }\end{array}$ \\
\hline Boletus edulis Bull.* (TBI5009295) & Datvis sok'o & Khortsa & 33 & Aug - Oct & Forest; Soil \\
\hline $\begin{array}{l}\text { Boletus erythropus Pers.* (TBI } \\
\text { 5009316) }\end{array}$ & $\begin{array}{l}\text { Ts'itelpekha } \\
\text { mukhisdzira }\end{array}$ & - & 33 & Aug-Nov & Primarily oak forest; Soil \\
\hline $\begin{array}{l}\text { Bovista nigrescens Pers.* } \\
\text { (TBI5009323) }\end{array}$ & Shavi pshuk'una & K'ok'oshol & 9 & Aug-Nov & Pastureland; Soil \\
\hline $\begin{array}{l}\text { Bovista plumbea Pers.* } \\
\text { (TBI5010007, TBI5010008, } \\
\text { TBI5009125, TBI5009126) }\end{array}$ & T'q'viisperi pshuk'una & K'ok'oshol & 9 & Aug-Nov & Pastureland; Soil \\
\hline $\begin{array}{l}\text { Calvatia candida (Rostk.) Hollós* } \\
\text { (TBI5010002) }\end{array}$ & Tetri tavak'a & Chazhi pu & 5 & Aug-Nov & Pastureland; Soil \\
\hline $\begin{array}{l}\text { Calvatia gigantea (Batsch) Lloyd* } \\
\text { (TBI5009322) }\end{array}$ & Giganturi pshuk'una & Chazhi pu & 4 & Jun-Sep & Subalpine meadow; Soil \\
\hline $\begin{array}{l}\text { Cantharellus cibarius Fr.* } \\
\text { (TBI5009301) }\end{array}$ & Miklio & $\begin{array}{l}\text { Miklio, Kat- } \\
\text { nmiskonai }\end{array}$ & 53 & Jun -Nov & $\begin{array}{l}\text { Primarily coniferous forest; } \\
\text { Soil }\end{array}$ \\
\hline $\begin{array}{l}\text { Chlorophyllum rachodes (Vittad.) } \\
\text { Vellinga (TBI5010040) }\end{array}$ & $\begin{array}{l}\text { Rbilobts'itela } \\
\text { ts'erosts'viva }\end{array}$ & - & 0 & Jul -Oct & Coniferous forest; Soil \\
\hline $\begin{array}{l}\text { Chroogomphus rutilus (Schaeff.) } \\
\text { O.K.Mill. (TBI5010036) }\end{array}$ & Ts'iteli lorts'ovana & - & 0 & Jun -Nov & Pine forest; Soil \\
\hline $\begin{array}{l}\text { Clitocybe nebularis (Batsch) P. } \\
\text { Kumm. (TBI5009309) }\end{array}$ & Mindvris sok'o & - & 0 & Aug-Nov & Forest edges; Soil \\
\hline $\begin{array}{l}\text { Clitopilus prunulus (Scop.) P. } \\
\text { Kumm. (TBI5009288) }\end{array}$ & Alublisdzira & - & 0 & Jun-Sep & $\begin{array}{l}\text { Primarily around Prunus } \\
\text { spp.; Soil }\end{array}$ \\
\hline $\begin{array}{l}\text { Coprinellus micaceus (Bull.) } \\
\text { Vilgalys, Hopple \& Jacq. Johnson } \\
\text { (TBI 5009337) }\end{array}$ & Mglis sok'o & - & 0 & May-Nov & $\begin{array}{l}\text { Broad-leaved forest; Soil } \\
\text { around stumps }\end{array}$ \\
\hline $\begin{array}{l}\text { Coprinopsis atramentaria (Bull.) } \\
\text { Redhead, Vilgalys \& Moncalvo* } \\
\text { (TBI 5009351) }\end{array}$ & Silio & Kokodil & 6 & May-Nov & $\begin{array}{l}\text { Grassland, deciduous forest; } \\
\text { Soil, buried dead wood }\end{array}$ \\
\hline $\begin{array}{l}\text { Cortinarius violaceus (L.) Gray } \\
\text { (TBI 5009361) }\end{array}$ & Lurjana & - & 0 & Jul -Oct & Forest; Soil \\
\hline $\begin{array}{l}\text { Craterellus cornucopioides (L.) } \\
\text { Pers. (TBI 5009333) }\end{array}$ & Dzabra sok'o & - & 0 & Aug-Nov & Primarily beech forest; Soil \\
\hline $\begin{array}{l}\text { Fistulina hepatica (Schaeff.) With. } \\
\text { (TBI 5009365) }\end{array}$ & Ghvidzla sok'o & - & 0 & Aug - Oct & $\begin{array}{l}\text { Primarily oak forest; Dead } \\
\text { wood }\end{array}$ \\
\hline $\begin{array}{l}\text { Flammulina velutipes (Curtis) } \\
\text { Singer (TBI 5009344) }\end{array}$ & Zamtris sok'o & - & 0 & $\begin{array}{l}\text { Sept } \quad- \\
\text { Dec }\end{array}$ & $\begin{array}{l}\text { Primarily on Populus; Dead } \\
\text { wood }\end{array}$ \\
\hline $\begin{array}{l}\text { Gomphidius glutinosus (Schaeff.) } \\
\text { Fr. (TBI 5009197) }\end{array}$ & $\begin{array}{l}\text { Nadzvnarebis } \\
\text { lorts'ovana }\end{array}$ & - & 0 & Aug - Oct & Primarily spruce forest; Soil \\
\hline $\begin{array}{l}\text { Hericium coralloides (Scop.) Pers. } \\
\text { (TBI 5009183) }\end{array}$ & Irmis bats'ara & - & 0 & Aug - Oct & $\begin{array}{l}\text { Primarily beech forest; Dead } \\
\text { wood }\end{array}$ \\
\hline $\begin{array}{l}\text { Hericium erinaceus (Bull.) Pers. } \\
\text { (TBI5010032) }\end{array}$ & Gula sok'o & - & 0 & Aug - Oct & Primarily forest; Dead wood \\
\hline $\begin{array}{l}\text { Hydnum repandum Fr. (TBI } \\
\text { 5009197) }\end{array}$ & Irmis tucha & - & 0 & Jul-Oct & Forest; Soil \\
\hline $\begin{array}{l}\text { Hygrophoropsis aurantiaca } \\
\text { (Wulfen) Maire (TBI 5009169) }\end{array}$ & Tsrumiklio & - & 0 & Jul-Oct & $\begin{array}{l}\text { Primarily coniferous forest; } \\
\text { Soil, decaying wood }\end{array}$ \\
\hline
\end{tabular}




\begin{tabular}{|c|c|c|c|c|c|}
\hline Scientific Name (Collection Nos.) & $\begin{array}{l}\text { Major Georgian } \\
\text { names according to } \\
\text { the literature }\end{array}$ & $\begin{array}{l}\text { Names } \\
\text { mentioned in } \\
\text { the interviews }\end{array}$ & $\begin{array}{l}\text { Numberof res- } \\
\text { pondents who } \\
\text { mentioned the species }\end{array}$ & Season & Habitat; Substrate \\
\hline $\begin{array}{l}\text { Kuehneromyces mutabilis (Schaeff.) } \\
\text { Singer \& A.H.Sm. (TBI 5009184) }\end{array}$ & $\begin{array}{l}\text { Zapkhulis } \\
\text { manch'k'vala }\end{array}$ & - & 0 & Jul-Nov & $\begin{array}{l}\text { Primarily beech forest; } \\
\text { Living or dead wood }\end{array}$ \\
\hline $\begin{array}{l}\text { Laccaria laccata (Scop.) Cooke } \\
\text { (TBI 5009156) }\end{array}$ & Martio & - & 0 & Jul-Nov & Forests; Soil \\
\hline $\begin{array}{l}\text { Lactarius deliciosus (L.) Gray* } \\
\text { (TBI 5009175) }\end{array}$ & Mch'ada & $\begin{array}{l}\text { Guiz, } \\
\text { Ch'vadvai }\end{array}$ & 63 & $\begin{array}{l}\text { Aug } \\
\text { Nov }\end{array}$ & Coniferous forest; Soil \\
\hline $\begin{array}{l}\text { Lactarius necator (Fr.) Karst (TBI } \\
\text { 5009161) }\end{array}$ & Shavi arq'a & - & 0 & Jul-Nov & $\begin{array}{l}\text { Primarily coniferous forest; } \\
\text { Soil }\end{array}$ \\
\hline $\begin{array}{l}\text { Lactarius piperatus (L.) Pers.* (TBI } \\
\text { 5009189) }\end{array}$ & Arq'a & Arq'ai & 82 & Jul-Nov & Primarily beech forest; Soil \\
\hline $\begin{array}{l}\text { Lactarius scrobiculatus (Scop.) Fr. } \\
\text { (TBI 5009162) }\end{array}$ & Q'viteli pach'ich'a & - & 0 & Jul-Oct & Forest; Soil \\
\hline $\begin{array}{l}\text { Lactarius torminosus (Schaeff.) } \\
\text { Gray (TBI 5009190) }\end{array}$ & Nabada sok'o & - & 0 & Jul-Nov & Primarily birch forest; Soil \\
\hline $\begin{array}{l}\text { Lactarius vellereus (Fr.) Fr. (TBI } \\
\text { 5009185) }\end{array}$ & Savartskhela & - & 0 & Jul-Oct & Forest; Soil \\
\hline $\begin{array}{l}\text { Lactarius volemus (Fr.) Fr. (TBI } \\
\text { 5009186) }\end{array}$ & Ch'ech'k'et'a & - & 0 & Jul-Oct & Primarily beech forest; Soil \\
\hline $\begin{array}{l}\text { Laetiporus sulphureus (Bull.) } \\
\text { Murrill (TBI5009177) }\end{array}$ & Q'viteli abeda & - & 0 & May - Oct & Forest; Living or dead wood \\
\hline $\begin{array}{l}\text { Leccinum aurantiacum (Bull.) Gray. } \\
\text { (TBI5009192) }\end{array}$ & Verkhvisdzira & - & 0 & Aug - Oct & Primarilypoplar stand; Soil \\
\hline $\begin{array}{l}\text { Leccinum scabrum (Bull.) Gray } \\
\text { (TBI5009193) }\end{array}$ & Arq'isdzira & - & 0 & Aug - Oct & Primarily beech forest; Soil \\
\hline $\begin{array}{l}\text { Lycoperdon perlatum Pers.* } \\
\text { (TBI5009067, TBI5009081) }\end{array}$ & $\begin{array}{l}\text { Khorklebiani } \\
\text { gudapshuk'a }\end{array}$ & Malatus & 6 & Jun-Nov & Forest, grassland; Soil \\
\hline $\begin{array}{l}\text { Lycoperdon pyriforme Schaeff.* } \\
\text { (TBI5009090) }\end{array}$ & $\begin{array}{l}\text { Mskhlisebri } \\
\text { gudapshuk'a }\end{array}$ & Malatus & 6 & Jun-Nov & $\begin{array}{l}\text { Various forests; Soil, } \\
\text { decaying wood }\end{array}$ \\
\hline $\begin{array}{l}\text { Macrolepiota excoriata (Schaeff.) } \\
\text { Wasser* (TBI5009304) }\end{array}$ & Tetri ts'erosts'viva & $\begin{array}{l}\text { Kama, } \\
\text { Irmispekha }\end{array}$ & 72 & Jun -Oct & $\begin{array}{l}\text { Various forests, roadsides; } \\
\text { Soil }\end{array}$ \\
\hline $\begin{array}{l}\text { Macrolepiota procera }(\text { Scop.) } \\
\text { Singer* (TBI 5009305) }\end{array}$ & Ts'erosts'viva & $\begin{array}{l}\text { Kama, } \\
\text { Irmispekha }\end{array}$ & 72 & Jun -Oct & $\begin{array}{l}\text { Various forests, roadsides, } \\
\text { fields; Soil }\end{array}$ \\
\hline $\begin{array}{l}\text { Marasmius oreades (Bolton) Fr. } \\
\text { (TBI5009287) }\end{array}$ & Jimla & - & 0 & May-Oct & Fields; Soil \\
\hline $\begin{array}{l}\text { Mycetinis alliaceus (Jacq.) Earle ex } \\
\text { A.W.Wilson \& Desjardin } \\
\text { (TBI5010036) }\end{array}$ & Sanelebela & - & 0 & May-Oct & $\begin{array}{l}\text { Various forest; Buried twigs } \\
\text { and other debris }\end{array}$ \\
\hline $\begin{array}{l}\text { Neolentinus lepideus (Fr.) Redhead } \\
\& \text { Ginns (TBI5009435) }\end{array}$ & Kertslovani pirpitk'bila & - & 0 & May-Oct & $\begin{array}{l}\text { Primarily coniferous forest; } \\
\text { Stumps }\end{array}$ \\
\hline Phallus impudicus L. (TBI5009416) & Kveq'nis gula & - & 0 & Aug - Oct & Primarily beech forest; Soil \\
\hline $\begin{array}{l}\text { Pleurotus cornucopiae (Paulet) } \\
\text { Rolland* (TBI 5009421) }\end{array}$ & Machalo & $\begin{array}{l}\text { Ts'iprash } \\
\text { tk'ubul, Ts'ipi } \\
\text { sok' }\end{array}$ & 61 & May-Oct & $\begin{array}{l}\text { Primarily beech forest; } \\
\text { Living or dead wood }\end{array}$ \\
\hline $\begin{array}{l}\text { Pleurotus ostreatus (Jacq.) } \\
\text { P.Kumm.* (TBI 5009449) }\end{array}$ & Khetamkhali & $\begin{array}{l}\text { Ts'iprash } \\
\text { tk'ubul, } \quad \text { Ts'ipi } \\
\text { sok' }\end{array}$ & 61 & May-Oct & $\begin{array}{l}\text { Primarily beech forest; } \\
\text { Living or dead wood }\end{array}$ \\
\hline $\begin{array}{l}\text { Polyporus squamosus (Huds.) Fr.* } \\
\text { (TBI5009436) }\end{array}$ & Dzerana & Dzero & 23 & May-Oct & $\begin{array}{l}\text { Primarily beech forest; } \\
\text { Living or dead wood }\end{array}$ \\
\hline $\begin{array}{l}\text { Psathyrella candolleana (Fr.) Maire } \\
\text { (TBI 5009422) }\end{array}$ & Tetri tsrumanch'k'vala & - & 0 & May-Oct & $\begin{array}{l}\text { Various forests; Living or } \\
\text { dead wood }\end{array}$ \\
\hline $\begin{array}{l}\text { Ramaria flava (Schaeff.) Quél.* } \\
\text { (TBI5010034) }\end{array}$ & Q'viteli sachechela & Irmis rka & 43 & Jun-Sep & Various forests; Soil \\
\hline $\begin{array}{l}\text { Russula adusta (Pers.) Fr. } \\
\text { (TBI5009418) }\end{array}$ & Mura sok'o & & 0 & Jun -Oct & $\begin{array}{l}\text { Primarily coniferous forest; } \\
\text { Soil }\end{array}$ \\
\hline $\begin{array}{l}\text { Russula aerugineaLindbl. ex Fr. } \\
\text { (TBI 5009447) }\end{array}$ & Mtredio & - & 0 & Jun -Oct & Primarily beech forest; Soil \\
\hline $\begin{array}{l}\text { Russula cyanoxantha (Schaeff.) Fr. } \\
\text { (TBI 5009433) }\end{array}$ & Ghvinio & - & 0 & Jul-Sep & Primarily beech forest; Soil \\
\hline Russula delica Fr. (TBI 5009426) & Tskhenis k'bila & - & 0 & Jun -Oct & Various forests; Soil \\
\hline $\begin{array}{l}\text { Russula emetica (Schaeff.) Pers. } \\
\text { (TBI 5009414) }\end{array}$ & Bghavana & - & 0 & Jun -Oct & $\begin{array}{l}\text { Primarily coniferous forest; } \\
\text { Soil }\end{array}$ \\
\hline $\begin{array}{l}\text { Russula foetens Pers. (TBI } \\
\text { 5009444) }\end{array}$ & Mq'rali khrashuna & - & 0 & Jun -Oct & Primarily birch forest; Soil \\
\hline Russula nigricans Fr. (TBI & Moshavo khrashuna & - & 0 & Jun -Oct & Various forest; Soil \\
\hline
\end{tabular}




\begin{tabular}{|c|c|c|c|c|c|}
\hline Scientific Name (Collection Nos.) & $\begin{array}{l}\text { Major Georgian } \\
\text { names according to } \\
\text { the literature }\end{array}$ & $\begin{array}{l}\text { Names } \\
\text { mentioned in } \\
\text { the interviews }\end{array}$ & $\begin{array}{l}\text { Numberof res- } \\
\text { pondents who } \\
\text { mentioned the species }\end{array}$ & Season & Habitat; Substrate \\
\hline \multicolumn{6}{|l|}{ 5009430) } \\
\hline Russula vesca Fr. (TBI 5009446) & Datuna & - & 0 & Jun -Oct & Deciduous forest; Soil \\
\hline $\begin{array}{l}\text { Russula virescens (Schaeff.) Fr. } \\
\text { (TBI 5009439) }\end{array}$ & Khakhvilo & - & 0 & Jun -Oct & Deciduous forest; Soil \\
\hline $\begin{array}{l}\text { Sarcodon imbricatus (L.) P.Karst. } \\
\text { (TBI5010035) }\end{array}$ & Irema sok'o & - & 0 & Aug - Oct & $\begin{array}{l}\text { Primarily coniferous forest; } \\
\text { Soil }\end{array}$ \\
\hline $\begin{array}{l}\text { Suillus luteus (L.) Roussel* (TBI } \\
\text { 5009424) }\end{array}$ & $\begin{array}{l}\begin{array}{l}\text { Chveulebrivi duma } \\
\text { sok'o }\end{array} \\
\end{array}$ & Khortsa soko & 33 & May - Oct & Primarily pine forest; Soil \\
\hline
\end{tabular}

* indicates the species mentioned in the interviews collected during the present study; Georgian names are given according to [25]; seasonality, habitat and substrate are given according to $[25,26]$.

\subsubsection{Mushroom Names}

Formerly the population of Svaneti must have had better knowledge of mushrooms, which is evidenced by literature data, in particular by a much higher number of local names in literature, in a number of cases accompanied by mushroom descriptions [11-17], than found in the current interviews (table 2). Reference [11] gives the highest number of vernacular names (30) with mushroom descriptions and in some cases descriptions of their use. The total number of vernacular names and their modifications in all the available dictionaries are about 50 . The majority of these names is now lost in the population; the following 12 vernacular names are still remembered: “Arq'ai", "Ch'aduai", "Chazhi pu”, "Dzero", "Guiz", "Guz", "K'ok'oshol", "Malatus", "Manch'k’varai", "Neq'v", "Ts'ipi sok' “, "Ts'iprash tk'ubul” (table 1). Three names: "Katmiskonai", "Irmispekha", "Irmis rka" mentioned by respondents were not found in any of the dictionaries considered. On the basis of mushroom descriptions provided by the above cited literature sources we managed to identify some of the species in question (table 2).

Table 2. Svanetian Names and Descriptions of Macro-fungi Given in the Literature and Species / Species Groups Identified for the Vernacular Names Presented (Chol. - Choluruli dialect, Lshkh. - Lashkhuri dialect, Lnt. - Lentekhuri dialect, Bz.- Balszemouri dialect, Bk. - Balskvemouri dialect, Zs. - group of Zemo Svanetian dialects).

\begin{tabular}{|c|c|c|}
\hline Svanetian name & $\begin{array}{l}\text { Latin name (identified } \\
\text { by the authors) }\end{array}$ & Fungi / lichen description in the references cited \\
\hline \multicolumn{3}{|l|}{ General names for fungi } \\
\hline Kokod (Chol.) & & Fungus [16] \\
\hline Kokood & & Fungus [16] \\
\hline Sik', T'q'ubul & & Fungus [12] \\
\hline Sok' & & Fungus [14] \\
\hline Sok'il (Lshkh.) & & Fungus [13] \\
\hline T'q'ubul & & Edible mushroom [27] \\
\hline T'q'ubul (Bz., Bk., Lshkh.) & & Fungus [14] \\
\hline T'q'ubul (Chol.) & & Edible mushroom [16] \\
\hline \multicolumn{3}{|l|}{ Polypores } \\
\hline Abed & Fomes sp. & Polypore [27] \\
\hline Abed (Chol.) & Fomes sp. & Boiled down fungus[16] \\
\hline Abed (Lshkh.) & Fomes sp. & $\begin{array}{l}\text { A hard fungus, grows out from fallen trees; the fungus is boiled down in ash and kneaded } \\
\text { to produce tinder [11] }\end{array}$ \\
\hline $\begin{array}{l}\text { Abed (Lshkh., Lnt.), Habed } \\
\text { (Bz.), Hobed (Bk.) }\end{array}$ & Fomes sp. & Polypore [14] \\
\hline $\begin{array}{l}\text { Abedissok'o, Hobediak, } \\
\text { Hobediakt'q'ubul }\end{array}$ & & Fomes fomentarius [17] \\
\hline Habed & Fomes sp. & Polypore[12] \\
\hline Ha-bed, Ho-bed & Fomes sp. & Polypore [15] \\
\hline Dzero & Polyporus squamosus & Eaten while fresh [11] \\
\hline Gak'asok' (Lshkh.) & Laetiporus sulphureus & $\begin{array}{l}\text { Walnut mushroom - yellow, non-edible [11]; walnut mushroom - yellowish, non-edible } \\
\text { [13] }\end{array}$ \\
\hline Gak'isok' (Chol.) & Laetiporus sulphureus & $\begin{array}{l}\text { Walnut mushroom, useful for tinder production after boiling down; like Mamali soko, } \\
\text { dry, used dry while treating "Dasuntkuli" (see explanation of "Dasuntkuli" in the text) }\end{array}$ \\
\hline
\end{tabular}




\begin{tabular}{|c|c|c|}
\hline & & with magic spells [16]; walnut mushroom [27] \\
\hline Ts'iva & Not identified & $\begin{array}{l}\text { Ts'iva is like Abedi, it gives tinder when boiled down, it easily flares up and does not die } \\
\text { out [11]; Ts'iva is like Abedi and it transforms to tinder when boiled down [14] }\end{array}$ \\
\hline Ts'iiva & Not identified & Tree fungus $[16]$ \\
\hline Puq'u (Bz.) & Not identified & Tree fungus used for tinder [14] \\
\hline \multicolumn{3}{|l|}{$\underline{\text { Agarics }}$} \\
\hline Arq'ai & Lactarius piperatus & White mushrooms with latex [16] \\
\hline Arq'ai (Chol.) & Lactarius piperatus & $\begin{array}{l}\text { White mushroom with white latex; poisonous when raw; its abundance betokened snowy } \\
\text { winter [16] }\end{array}$ \\
\hline Arq'ai (Lshkh.) & Lactarius piperatus & White, eaten roasted [11] \\
\hline Arq'ai (Lshkh.), Arq'a (Lnt.) & Lactarius piperatus & White mushroom, Arq'a mushroom; Arq'a mushroom is eaten roasted [14] \\
\hline Ch'aduai & Lactarius deliciosus & Red mushroom, eaten raw, roasted, boiled [11] \\
\hline Chazhisok' & Coprinus sp. & Grows on horse manure, non-edible [11] \\
\hline Ch'vadvai & Lactarius deliciosus & $\begin{array}{l}\text { Edible mushroom, appears in summer and persists till autumn. It this mushroom } \\
\text { predominated, people expected rich harvest [16] }\end{array}$ \\
\hline Guiz(Zs.), Guz (Lshkh.) & Lactarius deliciosus & Mch'ada soko [14] \\
\hline Kharik'bilai & Russula delica & Kharis k'bila - looks like Arq'a soko, is eaten roasted [11] \\
\hline Manch'k'varai & Armillaria mellea & Manch'k'vala [16] \\
\hline Manch'kvarai (Chol.) & Armillaria mellea & Manch'k'vala [16] \\
\hline Manch'k'varai (Lshkh.) & Armillaria mellea & $\begin{array}{l}\text { It is collected in spring, dried up and eaten in winter [11]; Manch'k'vala, collected in } \\
\text { summer, dried up and eaten in winter in soup [13] }\end{array}$ \\
\hline Neq'v & Amanita caesarea & Soft mushroom [11] \\
\hline T'q'ubla & $\begin{array}{l}\text { Pleurotus ostreatus, } P \text {. } \\
\text { cornucopiae }\end{array}$ & Edible mushroom, grows on trees [11] \\
\hline Ts'ipisok' & $\begin{array}{l}\text { Pleurotus ostreatus, } P \text {. } \\
\text { cornucopiae }\end{array}$ & Beech mushroom, edible [11] \\
\hline Ts'iprasht'q'ubul & & Pleurotus ostreatus [17] \\
\hline Vijik'orgol (Lashkh.) & Macrolepiota sp. & Grows on ground, looks like an umbrella, edible [11] \\
\hline \multicolumn{3}{|l|}{ Puffballs } \\
\hline Chazhipu & Bovista sp., Calvacia sp. & $\begin{array}{l}\text { Ts'khenis kuili - usual in riparian forest, rounded, small, becomes dusty when ripe. } \\
\text { Grows in fields, looks like cheese, crashes like "suluguni" }{ }^{3} \text { when weighed upon; } \\
\text { becomes dusty when ripe [11] }\end{array}$ \\
\hline K'ok'oshol (Chol.) & Bovista sp. & Small rounded good-looking fungus [16]; Fresh fungus[27] \\
\hline Malatus & & Lycoperdon [17] \\
\hline Malatus (Lshkh.) & Lycoperdon sp. & Meliis tsuili - grows on grass, white, later becomes black, edible while fresh [11] \\
\hline Mavlatus (Chol.) & Lycoperdon sp. & $\begin{array}{l}\text { Small white autumn fungi, after drying out it is full of black dust [16]; a fungus, which } \\
\text { becomes dust as soon as it grows up [27] }\end{array}$ \\
\hline \multicolumn{3}{|l|}{ Not identified } \\
\hline Q'ivnul sok' & & No other edible mushroom can be compared with it, good in March [11] \\
\hline K'orich'ol (Lshkh.) & & Blood red fungus, very small, good when roasted [11] \\
\hline K'oroch'ol (Lshkh.) & & White outside, blood red inside, very small, delicious when roasted [11] \\
\hline Mavsoq'ian sok' & & A fungus, which make one mad [11] \\
\hline Matkhep sok' (Lshkh.) & & Dapertkheba soko - spread over the ground, non-edible [11] \\
\hline Piluai & & A fungus, which make one mad [11] \\
\hline Tsaira sok' & & Elm fungus, black, edible, tasty [11] \\
\hline Tsats'khvi sok' & & Linden fungus, edible, tasty [11] \\
\hline Ghumuri sok' & & Spruce fungus, non-edible [11] \\
\hline Ghuninichv & & Khbostucha is good roasted; grows on pears and elsewhere [11] \\
\hline
\end{tabular}

\footnotetext{
${ }^{3}$ "Suluguni" is a kind of Georgian cheese.
} 


\begin{tabular}{|c|c|c|}
\hline Ghunitkhum & & Khbos tava, edible [11] \\
\hline Iebra sok' (Lshkh.) & & Sweet cherry tree fungus, red, non-edible [11] \\
\hline K'imeegh (Chol.) & & White fungus, non-edible [16] \\
\hline Kokodilar & & A good mushroom of middle size, red, tasty when roasted [11] \\
\hline \multicolumn{3}{|l|}{ Lichens } \\
\hline Chach & $\begin{array}{l}\text { Usnea spp., Bryoria spp., } \\
\text { Ramalina thrausta }\end{array}$ & Beard of a man and a tree[27] \\
\hline Pimpous & $\begin{array}{l}\text { Usnea spp., Bryoria spp., } \\
\text { Ramalina thrausta }\end{array}$ & Hair, tree moss $[11]$ \\
\hline
\end{tabular}

Several species mentioned in the interviews were not identified on account of insufficiently detailed descriptions and absence of specimen material; the vernacular names of these species are: "Datvisq'ura" (5 interviews), "Ts'itla" (3), and "Dedopala" (2).

The collective names for mushrooms in various dialects of Svanetian language are: "Sok", “T'q'ubul", "But'q'uli", "Kokod", "Sok'il", "Sik'"” (Table 2). At present the population mainly uses the word "Sok'o" for mushrooms in general. Species specific Svanetian mushroom names are thus clearly being lost in everyday life.

\subsubsection{Mushroom Oracles}

In Svaneti (in particular, Lashkheti, a historical commune of Svaneti) mushroom observations were formerly used as an oracle: it was thought that abundant "Ch'vadvai" (Lactarius deliciosus) promised good harvest, and much "Arq'ai" (Lactarius piperatus) betokened snowy winters [27]. None of the respondents interviewed mentioned/had heard about such folklore.

\subsubsection{Tinder}

Formerly tinder was made of "Abeda soko" (Fomes sp.) the name literally means "tinder mushroom". The population (mainly elders) still recalls how to prepare tinder. A birch polypore (Piptoporus betulinus (Bull.) P. Karst.) was thought to be the best material for tinder; it was well boiled with ash, well beaten, dried and ground, yielding tinder (B. Kakhberidze, Mest'ia, pers. comm., 2014). The younger population has already lost this knowledge.

\subsubsection{Mushrooms as Medicine}

The Svaneti population also used mushrooms for medicinal purposes. Almost $100 \%$ of the respondents collected already black (over-ripe) fruit-bodies of Lycoperdon and Bovista species from August to October, and kept them dry in glass pots during the whole year. The fungi were used to stop bleeding by pouring the contents of the fruit-bodies onto wounds; the powder was also used for treatment burns. The peel of a fruit-body was also put as a plaster on abscesses.

Another mushroom used for medicinal purpose is Lactarius deliciosus: a wine-like juice was produced by mixing this mushroom with sugar, and the juice was used for cough, and the treatment of diarrhea (D. Jinchradze, M. Kurasbediani and Sh. Kurasbediani, Babili, pers. comm., 2014). Several elders recalled that in their childhood their parents prepared a mushroom tincture, which was used by hunters to cure colds by rubbing the tincture onto body.

The medicinal use of fungi in Svaneti is also described in literature: "Patrik'i", "Patruki" was made of polypore and birch twigs; the twigs were sharpened like a pencil, the tip smeared with a mushroom based emollient and put into wound” [28]. “... „Mamali sok'o”, was used dry while treating "Dasuntkuli" with magic spells" [16]. "Dasunuli" is the same as "Dasuntkuli" - when (a snake) touches something with its mouth and poisons it" [29]; Georgian traditional medicine knows a category of rash, whose appearance is explained by taking food products passed over or smelled by a mouse, snake, etc. [30]; "Dasunva" means "to smell" and "Dasuntkva" means "to breathe on" in Georgian) According to various descriptions provided by the literature sources (Table 2), the polypore used must be Laetiporus sulphureus (Bull.) Murrill.

\subsection{Lichens}

Lichens, particularly, Usnea and Bryoria species as well as Ramalina thrausta (Ach.) Nyl.are called "Pimpa" or "Pimpous" [11] and "Chach" [27] in Svaneti. Lichens were never used for food there. "Pimpa" or "Pimpous", literally means "a man with dense hair and beard" and is also described as "conifer bristle." These species were used by hunters as field-bedding to keep warmth in cold winters. The use of lichens is reflected in local folklore: one of the respondents told a story about a hunter who went to Mt.Zhibelakhvi, killed a Caucasian goat, and when he broiled its meat, a giant ogre came to the cave where the hunter stayed. The ogre asked for the broiled meat. The hunter gave him a piece but when the ogre asked for another one, the hunter thought that his unexpected guest would eat the whole goat and thought to dodge the ogre. He took the "Pimpous", wrapped himself in lichens, and set fire to them with a burning log. The ogre did the same but burnt himself, rushed out of the cave and fell into an abyss (R. Pirtskhelani, Mest'ia, pers. comm., 2014).

\section{Conclusions}

The following major conclusions can be drawn from the results of the present work: the local population of Svaneti currently uses 22 species of mushrooms for food, 10 of which are used most frequently. In the past the population of Svaneti must have had better knowledge of mushrooms, which is 
evidenced by literature data, in particular by the much higher number of local names. Fungi were originally not only used for food, but also for tinder production (polypores), for medicine (sticks for emollient application (polypores), medications for internal and external use (agarics, puffballs)). A limited number of lichens were used by hunters as field-bedding to keep warm in the cold winters. The uses of fungi and lichens are clearly reflected in the local folklore. Further investigations are needed to (a) clarify the identity of certain fungi indicated by respondents by vernacular names or usage descriptions / found in the literature without any descriptions or with insufficient descriptions to allow a identification to species or species group, (b) to compare fungi use with other regions of Georgia and the Caucasus in general. It is greatly recommended to publish local popular guidebooks on edible (67 recorded vs. 22 actually used by the local population), and poisonous (many respondents mentioned frequent cases of Amanita poisoning because of confusion with Agaricus), mushrooms occurring in the study region, to be disseminated among the population.

\section{Acknowledgements}

The Georgian authors also would like to thank the Shota Rustaveli Natonal Science Foundation, Georgia, for funding the field trip to Svaneti within the framework of the research project "Ethnobiological study of fungi and lichens of Georgia".

We would like to thank Ms. Chato Gujejiani who helped us in translation of Svanetian descriptions of a number of fungi and all the people of Svaneti, especially the residents of the villages of Zemo and Kvemo Svaneti, who kindly agreed to participate in the ethnobiological interviews, as well as those who helped the authors in organizing the expedition.

\section{References}

[1] R. Singer, "Pilze aus dem Kaucasus 2. Ein Beitrag zur Flora Swanetzens und einiger angrenzender Taler," Botanischer Centralblatt Beihefte, vol. 48(2), pp. 513-542, 1931.

[2] I. G. Nakhutsrishvili, Agarics of Georgia. Tbilisi: Metsniereba, 1975.

[3] V. Kh. Gulmagarashvili, New species of Aphillophorales for Georgia. Reports of the Academy of Sciences of the Georgian SSR, vol. 91(3), pp. 685-687, 1978.

[4] T. Anchabadze, Materials for study of Discomicetes of Svaneti, Bulletin of the State Museum of Georgia, vol. XX-A, pp. 111-127, 1960.

[5] I. G. Nakhutsrishvili, Flora of Spore-producing Plants of Georgia (A Conspectus). Tbilisi: Metsniereba, 1986.

[6] E. Vainio, Lichenes in Caucaso et in Peninsula Taurica annis 1884-1885 ab H. Lojka et M. Dechy collecti, Termeszetr. Fuz., vol. 22, pp. 269-343, 1899.

[7] A. Jatta, Lichenes in Sommier S. et Levier E. Enumeratio plantarum anno 1890 in Caucaso lectorum. Acta Horti
Petropolitani, vol. XVI., 1900.

[8] M. Dechy, „Kaukasus". Reisen und Forschungen im kaukasischen Hochgebirge. Bd. III. Bearbeitung der gesammelten Materialien von F. Filarszky, E. Csiki, K. Papp, F. Schafarzi k und M. v. Dechy. Mit 36 Lichtdrucktafeln, Berlin: Dietrich Reimer (E. Vohsen), 1907.

[9] Degelius G., The Lichen genus Collema in Europe, Symb. Sot. Upsal., vol. 13, 1954, p. 2.

[10] O. Szatala, Lichenes in Peninsula Taurica et in Caucaso ab F. Komienski, D. Sosnowsky et E. Koenig collecti, Borbasia, vol. 4, pp. 70-96. 1944.

[11] A. Oniani, Collection of Svanetian Names of Trees and Shrubs (on Lashkuri Dialect), Materials on Japhetic linguistics, vol. VIII. Petrograd: Academy of Sciences Press, 1917.

[12] B. Nizharadze, Georgian-Svanetian-Russian Dictionary, Balszemouri dialect. Tbilisi: Universali, 2012.

[13] K. Dondua, Svanetian-Georgian-Russian dictionary (Lashkhuri Dialect). Tbilisi: Publishing house of Sulkhan-Saba Orbeliani Pedagogical University of Tbilisi, 2001.

[14] V. Topuria, Svanetian Dictionary. Tbilisi: Kartuli Ena, 2000.

[15] N. Ia. Marr, Extract from Svanetian-Russian Dictionary, Materials on Japhetic linguistics, vol. X. Petrograd: Russian State Academy Press, 1922.

[16] A. Liparteliani, Svanetian-Georgian Dictionary (Choluruli sub-dialect). Tbilisi, 1994.

[17] A. Makashvili, Botanical Dictionary. Tbilisi: Metsniereba, 1991.

[18] D. Ukleba, For physical-geographic characteristic of Zemo Svaneti, Manuscript of Candidate Dissertation, TSU, 1952, unpublished.

[19] R. Gagnidze and M. Davitadze, The Local Flora [Plant World of Georgia]. Batumi: GamomtsemlobaAch'ara, 2000.

[20] Major results of the first national general census of the population of Georgia (Population of villages of Georgia), vol. 2, T. Beridze, I. Archvadze and Ts. Eliadze, Eds. Tbilisi: State Department of Statistics of Georgia, 2003, pp. 143-146, 164-169,

[21] M. Kordzakhia and Sh. Javakhishvili, Climate of Georgia. Tbilisi: Ganatleba, 1971.

[22] N. Zazanashvili, R. Gagnidze and G. Nakhutsrishvili, Main types of vegetation zonation on the mountains of the Caucasus, Acta Phytogeographica Suecica, vol. 85, pp. 7-16, 2000.

[23] V. Topuria, Svanetian language (1965), Unpublished works on Svanetian language, in Works, vol. 2, V. Topuria. Tbilisi: Kartuli ena, 2002, pp. 150-219.

[24] P. M. Kirk, Species Fungorum (version Feb 2014), in Species 2000 \& ITIS Catalogue of Life, 22nd December 2014, Y. Roskov, L. Abucay, T. Orrell, D. Nicolson, T. Kunze, A. Culham, N. Bailly, P. Kirk, T. Bourgoin, R.E. DeWalt, W. Decock and A. De Wever, Eds. Digital resource at www.catalogueoflife.-org/col. Naturalis, Leiden, the Netherlands: Species 2000, 2014. 
[25] I. Nakhutsrishvili, Fungi of Georgia. Tbilisi: BunebaPrinti, 2007.

[26] K. Kanchaveli, I. Nakhutsrishvili and Tz. Inashvili, Key to Spore-producing Plants of Georgia, vol. 1. Tbilisi: Metsniereba, 1981.

[27] A. Liparteliani, Svanetian-Georgian Dictionary (Choluruli Sub-dialect). Tbilisi: Diogene, 2014.
[28] M. Burduli, Folk medicine in West Georgia (according to Svanetian Ethnographic Materials). Akhaltsikhe: Akhaltsikhe University, 2010.

[29] Explanatory Dictionary of the Georgian Language, A. Chikobava, Ed.-in-Chief. Tbilisi: Main Editorial Board of the Georgian Soviet Encyclopedia, 1986.

[30] Gurian Medical Book, J. Kharchilava, Comp., M. Asatiani Ed. Tbilisi: Pergamenti, 1993. 